OPEN ACCESS

Edited by:

Shuozhi Xu,

University of California, Santa Barbara,

United States

Reviewed by:

Yifei Zeng,

ExxonMobil, United States

Akshay Dandekar,

Stress Engineering Services, Inc.

United States

*Correspondence:

Shun Hu Zhang

shzhang@suda.edu.cn

Specialty section:

This article was submitted to Computational Materials Science,

a section of the journal

Frontiers in Materials

Received: 14 July 2021 Accepted: 08 September 2021 Published: 20 September 2021

Citation:

Zhang SH, Xin JL and Che LZ (2021) Modeling of Rolling Force for Thick Plate of Multicomponent Alloys and Its Application on Thickness Prediction.

Front. Mater. 8:741144.

doi: 10.3389/fmats.2021.741144

\section{Modeling of Rolling Force for Thick Plate of Multicomponent Alloys and Its Application on Thickness Prediction}

\author{
Shun Hu Zhang*, Jia Lin Xin and Li Zhi Che \\ Shagang School of Iron and Steel, Soochow University, Suzhou, China
}

During the rolling process of thick plate, the nonlinear specific plastic power that derived from the non-linear Mises yield criterion is difficult to be integrated, which has restricted the establishment of a rolling force model. To solve this problem, a new yield criterion is firstly established, and then used to derive a linear specific plastic power. Meanwhile, a kinematically admissible velocity field whose horizontal velocity component obeys the Logistic function is proposed to describe the metal flow of the deformed plate. On these bases, the rolling energy items including the internal deformation power of the deformed body, friction power on the contact surface, and shear power on the entry and exit sections are integrated successively, and the rolling force model is established. It is proved that the model can predict the rolling force well when compared with the actual data of multicomponent alloys. Besides, the formula for predicting the outlet thickness is ultimately given upon this derived model, and a good agreement is also found between the predicted values and the actual ones, since the absolute errors between them are within $0.50 \mathrm{~mm}$.

Keywords: rolling force, yield criterion, specific plastic power, thickness prediction, multicomponent alloys

\section{HIGHLIGHTS}

- A linear specific plastic power is derived form a linear yield criterion, which can be used to solve the nonlinear integral problem.

- A velocity field that was constructed in the form Logistic function can describe the metal flow in the deformation zone well.

- A good prediction of outlet thickness can be obtained through the present rolling force model due to its high predictive accuracy.

\section{INTRODUCTION}

The yield behavior of a material can be described by a yield criterion. It is an important basis for judging whether a material undergoes plastic deformation, or taken as an mechanical equation that must be solved for obtaining the required force for material forming. So far, there are many yield criteria, which are generally expressed in the form of stresses. In 1864, Tresca proposed the maximum shear stress theory (Tresca, 1864), which provided a basic theoretical explanation for the yield characteristics of materials. In 1900, Mohr used the Coulomb's formula of shearing strength for rock and soil to improve his own strength theory and published the Mohr-Coulomb criterion (Mohr, 1900). This criterion is indeed a modification of the Tresca's theory, in which the maximum 
shear stress is no longer a constant, but a function of the normal stress in the same plane. In 1913, von Mises believed that if the secondary invariant of deviation stress reached a specific value, then the metal would turn from elastic deformation to plastic deformation (Mises, 1913; Mises, 1928). Based on this assumption, the Mises yield criterion was proposed. However, the mathematical form of this criterion is nonlinear, which is not convenient for the derivation of analytical solutions of material forming force, such as the rolling force. In 1951, Drucker and Prager (Drucker and Prager, 1952) added the influence of hydrostatic pressure to the Mises' formula, and the yield surface is shown to be a conical surface. Yu et al. (Yu, 2002) established the twin shear stress (TSS) yield criterion in 1961, and modified it in 1985 (Yu et al., 1985). The twin shear stress yield criterion assumes that when the sum of two larger principal shear stresses reaches a critical value, the material yields. This criterion is usually used to predict the upper limit of mechanical parameters. In 2005, Yu et al. (Gao and Yu, 2005) believed that although the TSS yield criterion can account for the influence of the intermediate principal stress, it neglected the influence of the minimum shear stress on the material yielding, and then they proposed the triple-shear stress yield criterion. In the case of combined shear stress on the element body, the threeshear yield criterion is more consistent with the actual results than the single-shear or twin shear yield criteria.

By summarizing the above information, it can be found that the Tresca yield criterion brings in a lower limit solution, the twin shear stress yield criterion brings in an upper limit solution. Although the nonlinear Mises yield criterion has high accuracy, it is difficult to obtain the analytical solution of material forming force. For this reason, it is necessary to find a new linear yield criterion whose locus can lies in between those based on the Tresca yield criterion and the twin shear stress yield criterion, and can approximate the Mises circle well, in order to meet the requirements of high accuracy and ease of calculation.

Thick plate is widely used in construction machinery, oil pipeline, offshore platform and other structural load-bearing part. How to accurately obtain the rolling force and rolling torque of thick plate is related to the selection of production parameters. The difficulty in solving the nonlinear Mises plastic power must be solved in the modeling of rolling force. In 2000, Wang et al. (Wang, 2000) used the three-dimensional stream function method to analyze the plastic processing problem, and established a flat roll rolling model considering the widening. Jia et al. (Jia et al., 2016) used the Zener-Hollomon parameter method to establish a flow stress model during unidirectional compression, and also established a flow stress model for variable temperature rolling. Liu (Liu, 2017) proposed a new exponential velocity field that can reflect the change law of metal flow for finishing rolling passes. Li et al. (Li and Wang, 1996) used the Hill yield criterion to study the stress field and velocity field of the plane stress problem of orthotropic materials, and obtained the result that the characteristic lines of the stress field and velocity field overlap everywhere. Zhang et al. (Zhang et al., 2019) proposed a new type of velocity field considering the rolling permeability to analyze the rolling of extra-thick plates. Their results show that the rolling force and rolling torque models considering the deformation penetration can give more accurate results. Based on the above researches, it can be found that the establishment of the velocity field by the analytical method can be used well in analyzing the problem of thick plate rolling, and is beneficial to the optimization of rolling parameters. However, the existing velocity fields with the above function form fail to accurately describe the vertical flow characteristic, which can arise big errors in predicting rolling force and torque. One important reason is that most of the available velocity fields can just satisfy the velocity boundary condition on the inlet section and neglect the required condition on the outlet section. In fact, a good velocity should satisfy the velocity boundary conditions on the inlet section and the outlet section simultaneously. Quantitative assessment is necessary for proposing a reasonable velocity field, which is desired to be done in the future.

In order to solve the above problems, i.e. the problem of integral difficulty of rolling power and the problem of inefficient accuracy of available models, this paper intends to develop a linear yield criterion that can approximate the nonlinear Mises yield criterion well, and bring in a linear specific plastic power to analyze the plate rolling process. For this purpose, a new velocity field in the form of Logistic function, that can satisfy the velocity boundary conditions rigorously, is also proposed for describing the thick plate rolling. Based on the new yield criterion and the new velocity field, the corresponding internal deformation power, friction power and shear power are obtained, and then the analytical rolling torque and force are obtained through the variational method. Meanwhile, the bouncing equation is given to predict the thickness of the rolled plate by embedding the rolling force model, and a comparison is carried out between the calculated ones and the experimental data.

\section{DERIVATION OF AVERAGE SLOPE YIELD CRITERION}

\section{Yield Criterion Expression}

The locus of the Mises yield criterion is a circle on the $\pi$ plane. The dodecagon between the circumscribed hexagon (TSS yield locus) and the inscribed hexagon (Tresca yield locus) can be used as a linear approximation to the Mises circle. In Figure 1, suppose that there is a moving point $E$ on the line segment $B F$, and a new yield edge can be obtained by connecting $B^{\prime} E$. Set the angle $\angle F B^{\prime} E$ between $B^{\prime} F$ and $B^{\prime} E$ to $\theta$, then it can be seen that when $\theta=0^{\circ}$, it corresponds to Tresca yield locus $B^{\prime} F$ and when $\theta=30^{\circ}$, it corresponds to the TSS yield locus $B^{\prime} B$.

In Figure 1, the radius of the Mises circle satisfies $O B^{\prime}=O D=$ $\sqrt{6} / 3 \sigma_{s}$ ( $\sigma_{s}$ is the yield strength). So, according to the geometric relationship, $O F=\sigma_{s} / \sqrt{2}, B^{\prime} F=\sigma_{s} / \sqrt{6}$.

Set the origin of the coordinate system at the point $B^{\prime}$, as shown in Figure 2, the slope of $B^{\prime} E$ is

$$
k(\theta)=\tan \theta, 0^{\circ} \leq \theta \leq 30^{\circ} .
$$

From the mean value theorem of integral (Zhang et al., 2020), the mean slope $\bar{k}$ of $B^{\prime} E$ can be obtained as 


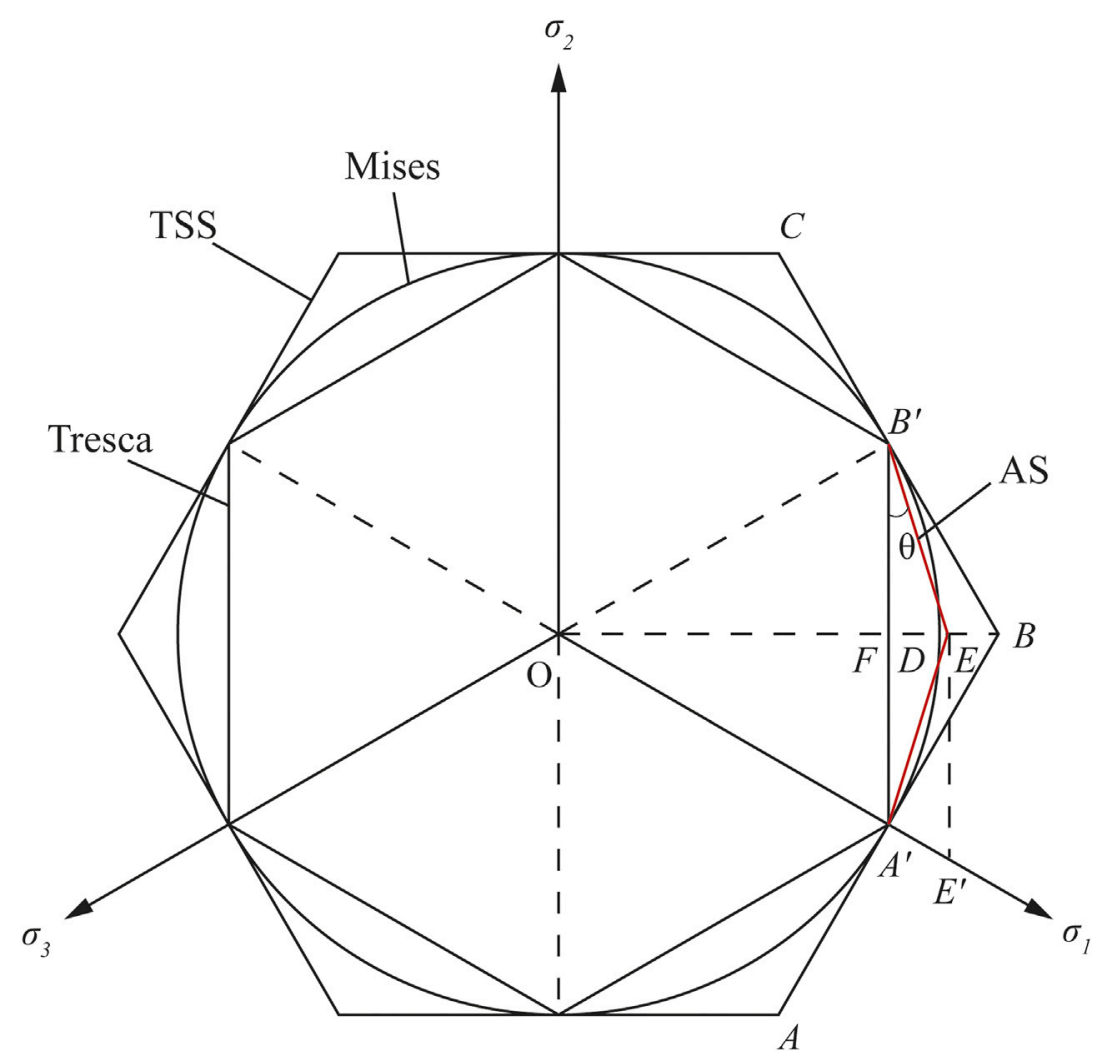

FIGURE 1 | Different loci on the $\pi$-plane.

$$
\bar{k}=\frac{1}{\left(\frac{\pi}{6}-0\right)} \int_{0}^{\frac{\pi}{6}} \tan \theta d \theta=0.275
$$

Let $\bar{k}=\tan \theta$, the undetermined value of $\theta$ and the length of $B^{\prime} E$ at this time can be obtained

$$
\begin{gathered}
\theta=\arctan \bar{k}=0.268 \\
B^{\prime} E=\frac{B^{\prime} F}{\cos \theta}=0.423 \sigma_{s}
\end{gathered}
$$

Based on the above formulas, we can get

$$
F E=\sqrt{B^{\prime} E^{2}-B^{\prime} F^{2}}=0.110 \sigma_{s} .
$$

The modulus length of the deviation vector on the Mises yield locus is

$$
O D=O B^{\prime}=\sqrt{6} / 3 \sigma_{s} .
$$

The modulus length of the deviation vector of the newly proposed yield criterion is

$$
O E=0.817 \sigma_{s}
$$

So, the error between them is

$$
\Delta=\left(0.817-\frac{\sqrt{6}}{3}\right) / \frac{\sqrt{6}}{3}=0.0617 \% \text {. }
$$

It can be seen that the vector modulus length of the newly proposed yield criterion is just a little higher than that of the Mises yield criterion, which means that the point $E$ should be locates in between $B$ and $D$, and near $D$, as shown in Figure 2.

The expressions of the lines $A^{\prime} E$ and $B^{\prime} E$ are established in the following section. Figure 3 shows the projection of the principal stress component $\sigma_{1}$ on the $\pi$ plane. Considering the projection relationship and the plain deformation condition, the three principal stress components $\sigma_{1}, \sigma_{2}, \sigma_{3}$ at point $E$ can be calculated by

$$
\left\{\begin{array}{l}
\sigma_{1}=\frac{O E \times \sqrt{3}}{\sqrt{2} \cos 30^{\circ}}=1.1554 \sigma_{s} \\
\sigma_{3}=0 \\
\sigma_{2}=\frac{\sigma_{1}+\sigma_{3}}{2}=0.57776 \sigma_{s} .
\end{array}\right.
$$

It can be assumed here that the $A^{\prime} E$ line can be written in the following form

$$
\sigma_{1}-a_{1} \sigma_{2}-a_{2} \sigma_{3}-c=0
$$

When a material yields, there have $c=\sigma_{s}, a_{1}+a_{2}=1$. Substituting Eq. 9 into Eq. 10, it produces

$$
a_{1}=0.268, a_{2}=0.732 \text {. }
$$




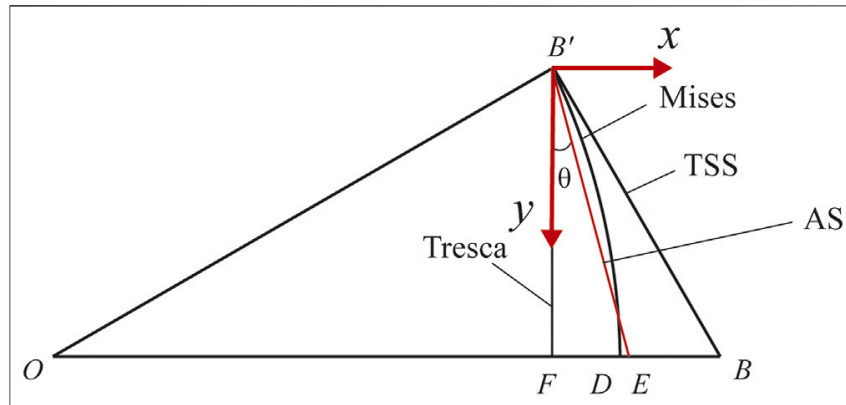

FIGURE 2 | The geometry of the average slope yield criterion.

Substituting Eq. 11 into Eq. 10, the expression of $A^{\prime} E$ can be derived as

$$
\sigma_{1}-0.268 \sigma_{2}-0.732 \sigma_{3}=\sigma_{s}, \sigma_{2} \leq \frac{1}{2}\left(\sigma_{1}+\sigma_{3}\right) .
$$

In the same way, the equation of the locus $B^{\prime} E$ can be determined as

$$
0.732 \sigma_{1}+0.268 \sigma_{2}-\sigma_{3}=\sigma_{s}, \sigma_{2} \geq \frac{1}{2}\left(\sigma_{1}+\sigma_{3}\right) .
$$

Eqs 12, 13 are the mathematical expressions of the new yield criterion, which is a linear combination of the principal stress components. Because the slope of the locus side $B^{\prime} E$ of this criterion is the mean value of the slopes of the Tresca and TSS loci, it can be called as the average slope yield criterion.

It can be seen from Figure 2 that the present yield locus intersects the Mises circle, and the vertex angles are as follows:

$$
\left\{\begin{array}{l}
\angle F B^{\prime} E=\angle \theta=15.355^{\circ} ; \\
\angle O B^{\prime} E=60^{\circ}+15.355^{\circ}=75.355^{\circ} ; \\
\angle O E B^{\prime}=180^{\circ}-30^{\circ}-75.355^{\circ}=74.645^{\circ} \\
2 \angle O B^{\prime} E=150.71^{\circ}, 2 \angle O E B^{\prime}=149.29^{\circ}
\end{array}\right.
$$

Figure 1 and Eq. 14 show that the present yield locus is an equilateral and non-equiangular dodecagon which intersects the Mises circle with twelve intersection points. Of which, six vertices of the locus are on the Mises circle, and the vertex angle is $150.71^{\circ}$. The other six vertices are located outside the Mises circle, with a distance of $0.000503 \sigma_{s}$ and the vertex angle is $149.29^{\circ}$. Except for these, it is known that each side length of the present dodecagon is equal to $0.423 \sigma_{s}$.

\section{Specific Plastic Power}

Suppose that the stress component $\sigma_{i j}$ satisfies $f\left(\sigma_{i j}\right)=0$ and the strain rate component $\dot{\varepsilon}_{i j}$ satisfies the flow rule of plastic deformation, then we can have (Kobayashi et al., 1989)

$$
\dot{\varepsilon}_{i j}=\mathrm{d} \lambda \frac{\partial f}{\partial \sigma_{i j}}=d \lambda \sigma_{i j}^{\prime} .
$$

Assuming that there are $\lambda \geq 0, \mu \geq 0$, then from Eqs 12, 15, it results in

$\dot{\varepsilon}_{1}: \dot{\varepsilon}_{2}: \dot{\varepsilon}_{3}=1:(-0.268):(-0.732)=\lambda:(-0.268) \lambda:(-0.732) \lambda$. (16)

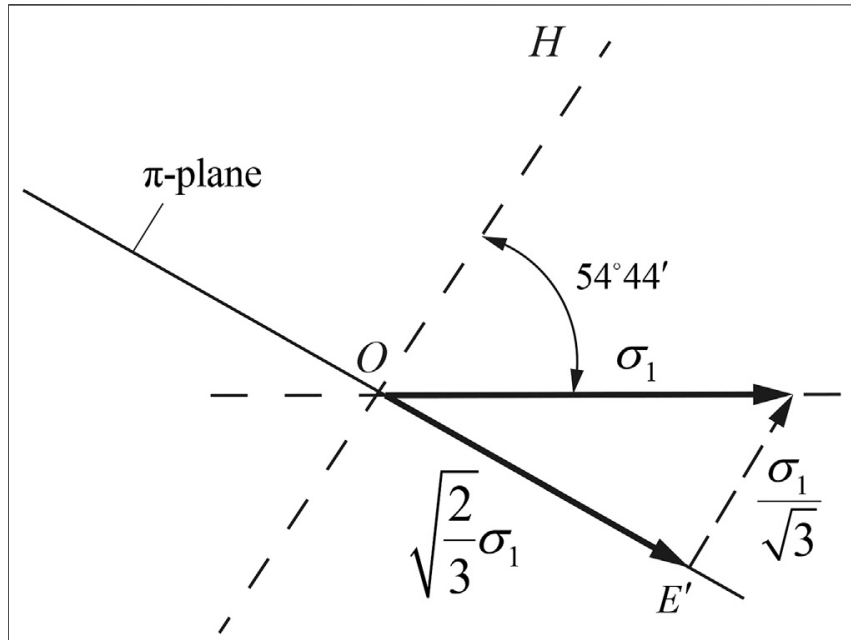

FIGURE 3 | The locus of $\sigma_{1}$ on the $\pi$-plane.

Similarly, according to Eqs 13, 15, it results in

$\dot{\varepsilon}_{1}: \dot{\varepsilon}_{2}: \dot{\varepsilon}_{3}=0.732: 0.268:(-1)=0.732 \mu: 0.268 \mu:(-\mu)$.

The linear combination of the results obtained from the above two formulas gives

$\dot{\varepsilon}_{1}: \dot{\varepsilon}_{2}: \dot{\varepsilon}_{3}=(\lambda+0.732 \mu): 0.268(\mu-\lambda):(-0.732 \lambda-\mu)$.

Take $\dot{\varepsilon}_{1}=\lambda+0.732 \mu$, then

$$
\dot{\varepsilon}_{2}=0.268(\mu-\lambda), \dot{\varepsilon}_{3}=-(0.732 \lambda+\mu) .
$$

Since $\dot{\varepsilon}_{\text {max }}=\dot{\varepsilon}_{1}, \dot{\varepsilon}_{\text {min }}=\dot{\varepsilon}_{3}$, it results in

$$
\dot{\varepsilon}_{\max }-\dot{\varepsilon}_{\min }=1.732(\lambda+\mu),(\lambda+\mu)=\frac{1000}{1732}\left(\dot{\varepsilon}_{\max }-\dot{\varepsilon}_{\min }\right) .
$$

At the vertex $E$, we notice that $\sigma_{2}=\left(\sigma_{1}+\sigma_{3}\right) / 2$, so it can be obtained from Eqs 12, 13 that

$$
\sigma_{1}-\sigma_{3}=\frac{2000}{1732} \sigma_{s}
$$

From Eqs 20, 21, the specific plastic power calculated by $D\left(\dot{\varepsilon}_{i j}\right)=\sigma_{1} \dot{\varepsilon}_{1}+\sigma_{2} \dot{\varepsilon}_{2}+\sigma_{3} \dot{\varepsilon}_{3}$ (Hosford and Caddell, 2011) based on the present yield criterion can be obtained as

$$
\begin{aligned}
D\left(\dot{\varepsilon}_{i j}\right)=\sigma_{1} \dot{\varepsilon}_{1} & +\frac{\sigma_{1}+\sigma_{3}}{2} \dot{\varepsilon}_{2}+\sigma_{3} \dot{\varepsilon}_{3} \\
& =0.8660\left(\sigma_{1}-\sigma_{3}\right)(\mu+\lambda) \\
& =\frac{1732}{2000} \times \frac{2000}{1732} \sigma_{s} \times \frac{1000}{1732}\left(\dot{\varepsilon}_{\max }-\dot{\varepsilon}_{\min }\right) \\
& =\frac{1000}{1732} \sigma_{s}\left(\dot{\varepsilon}_{\max }-\dot{\varepsilon}_{\min }\right) \\
& =0.577 \sigma_{s}\left(\dot{\varepsilon}_{\max }-\dot{\varepsilon}_{\min }\right)
\end{aligned}
$$

By using the same method, the specific plastic power based on the Tresca yield criterion and the TSS yield criterion can be written as follows (Kobayashi et al., 1989) 


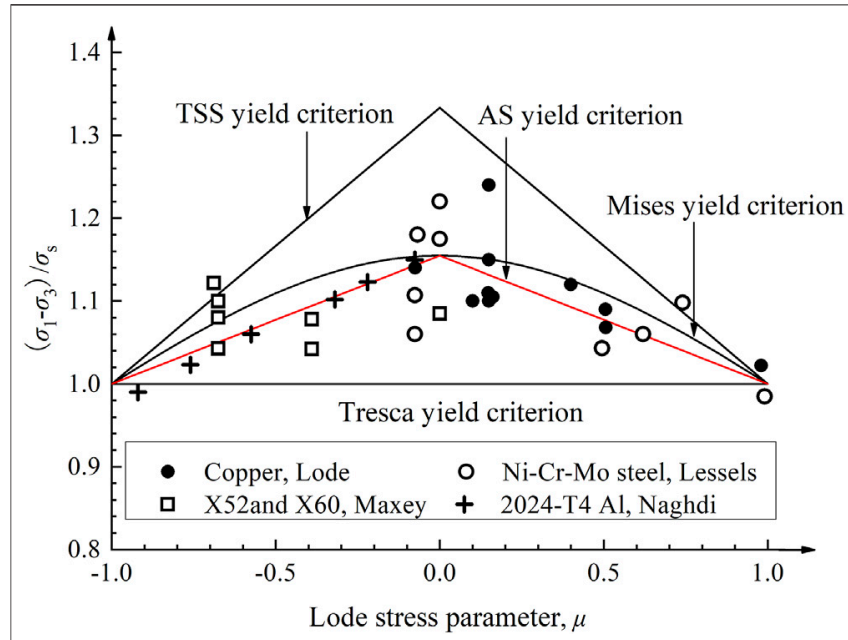

FIGURE 4 | Comparison of various yield criteria and experimental data.

$$
\begin{gathered}
D\left(\dot{\varepsilon}_{i j}\right)_{\text {Tresca }}=\frac{2}{3} \sigma_{s}\left(\dot{\varepsilon}_{\max }-\dot{\varepsilon}_{\min }\right), \\
D\left(\dot{\varepsilon}_{i j}\right)_{\text {TSS }}=\frac{1}{2} \sigma_{s}\left(\dot{\varepsilon}_{\max }-\dot{\varepsilon}_{\min }\right) .
\end{gathered}
$$

Comparing Eq. 22 with Eqs 23, 24, the relative errors of the specific plastic power among the present one, the Tresca one, and the TSS one are

$$
\left\{\begin{array}{l}
\Delta_{\text {Tresca }}=\left(0.577-\frac{1}{2}\right) / \frac{1}{2}=15.40 \% \\
\Delta_{\mathrm{TSS}}=\left(0.577-\frac{2}{3}\right) / \frac{2}{3}=-13.45 \%
\end{array}\right.
$$

The relative errors in Eq. 25 indicate that the calculated value of using the mean slope yield criterion is $15.40 \%$ higher than the calculated value based on the Tresca one, but is lower $13.45 \%$ than that based on the TSS one.

\section{Experimental Validation}

When the principal stress components satisfy $\sigma_{1} \geq \sigma_{2} \geq \sigma_{3}$, Lode proposed a stress characteristic parameter to reflect the difference among different yield criteria. The parameter is (Lode, 1926):

$$
\mu=\frac{2 \sigma_{2}-\sigma_{1}-\sigma_{3}}{\sigma_{s}} .
$$

Substituting Eq. 26 into the Tresca criterion, Mises criterion, TSS yield criterion as well as the AS yield criterion, the following expressions in terms of the Lode characteristic parameter can be derived as follows:

Tresca:

$$
\frac{\sigma_{1}-\sigma_{3}}{\sigma_{s}}=1
$$

Mises:

$$
\frac{\sigma_{1}-\sigma_{3}}{\sigma_{s}}=\frac{2}{\sqrt{3+\mu^{2}}}
$$

TSS:

$$
\frac{\sigma_{1}-\sigma_{3}}{\sigma_{s}}=\left\{\begin{array}{l}
\frac{4+\mu}{3},-1 \leq \mu \leq 0 \\
\frac{4-\mu}{3}, 0 \leq \mu \leq 1
\end{array}\right.
$$

AS:

$$
\frac{\sigma_{1}-\sigma_{3}}{\sigma_{s}}=\left\{\begin{array}{l}
\frac{2000+268 \mu}{1732},-1 \leq \mu \leq 0 \\
\frac{2000-268 \mu}{1732}, 0 \leq \mu \leq 1 .
\end{array}\right.
$$

Based on the above expressions, a comparison can be carried out with the experimental data from Ref. (Lode, 1926; Lessells and Macgregor, 1940; Naghdi et al., 1957; Maxey, 1974), shown in Figure 4.

It can be seen from Figure $\mathbf{4}$ that the Tresca yield criterion predicts the lower bound of the experimental data, while the TSS yield criterion predicts the upper bound. The present calculated value is lie between those based on the TSS criterion and the Tresca criterion, and closes to that based on the Mises yield criterion. It can be seen that the predictive results based on the AS yield criterion correlate well with the experimental data, and can bring in the reasonable intermediate results since both the average error and the maximum error are very small, just 3.21 and $10.66 \%$ respectively.

\section{MECHANICAL ANALYSIS OF THICK PLATE ROLLING \\ Velocity Field in the Form of Logistic Function}

As shown in Figure 5, $h_{0}$ is half thickness of the workpiece on the inlet section, $h_{1}$ is half thickness of the workpiece on the outlet section, $R$ is the radius of the roller, point $O$ is the geometrical center of the roller, $v_{0}$ is the initial velocity on the inlet section, and $v_{1}$ is the final velocity on the outlet section. $\theta$ is the contact angle, $\alpha$ is the neutral angle, $x$ is the length of the neutral point from the entrance of the deformation zone, and $h_{x}$ is half thickness of the workpiece in the deformation zone. It should be noted that when the tangential velocity of the rolled workpiece at the neutral point is equal to the tangential velocity of the roller, and the tangential velocity discontinuity and friction power at this time are both zero. According to the geometric relationship in Figure 5, the main deformation parameters can be expressed as (Li et al., 2017)

$$
\left\{\begin{array}{l}
z=h_{x}=R+h_{1}-\left[R^{2}-(l-x)^{2}\right]^{1 / 2} \\
z=h_{\alpha}=R+h_{1}-R \cos \alpha .
\end{array}\right.
$$

where, $h_{x}$ is the half thickness at the position $x$ and $h_{\alpha}$ is the corresponding thickness at the angle $\alpha$.

$$
\begin{gathered}
l-x=R \sin \alpha, \mathrm{d} x=-R \cos \alpha \mathrm{d} \alpha \\
h_{x}^{\prime}=-\tan \alpha, h_{x}^{\prime \prime}=\left(R \cos ^{3} \alpha\right)^{-1}
\end{gathered}
$$




$$
\left\{\begin{array}{l}
y=b_{x}=b_{0}+\frac{\Delta b}{l} x=b_{1}-\frac{\Delta b}{l} R \sin \alpha \\
b_{x}^{\prime}=\frac{\Delta b}{l} .
\end{array}\right.
$$

From Eqs 31-34, the boundary conditions are as follows

$$
\left\{\begin{array}{l}
x=0, \alpha=\theta: h_{x}=h_{\alpha}=h_{\theta}=h_{0}, b_{x}=b_{\alpha}=b_{\theta}=b_{0}, h_{x}^{\prime}=-\tan \theta, b_{x}^{\prime}=-\frac{\Delta b}{l} R \cos \theta ; \\
x=l, \alpha=0: h_{x}=h_{\alpha}=h_{1}, b_{x}=b_{\alpha}=b_{1}, h_{x}^{\prime}=0, b_{x}^{\prime}=0 .
\end{array}\right.
$$

where, $b_{x}$ is the half of plate width along the $x$ and $\Delta b=b_{1}-b_{0}$ is the spread between the final width and the initial width.

This article assumes that the horizontal velocity component $v_{x}$ in the deformation zone increases gradually according to the Logistic function from the inlet to the outlet, that is, it has the following functional form

$$
v_{x}=\frac{c_{1} c_{3} \mathrm{e}^{c_{2} x}}{c_{1}+c_{3}\left(\mathrm{e}^{c_{2} x}-1\right)} .
$$

where, $c_{1}, c_{2}, c_{3}$ are undetermined parameters, which are determined by the boundary conditions on the entrance and exit sections: $x=0, v_{x}=v_{0} ; x=l / 2, v_{x}=\left(v_{0}+v_{1}\right) / 2 ; x=l, v_{x}=$ $v_{1}$. So, the following velocity field can be established

$$
\left\{\begin{array}{l}
v_{x}=\frac{\left(v_{0}+v_{1}\right)}{1+\left(\frac{v_{1}}{v_{0}}\right)^{1-\frac{2 x}{l}} ;} \\
v_{y}=b_{x}^{\prime} \cdot v_{x}=\frac{\Delta b}{l} \cdot \frac{\left(v_{0}+v_{1}\right)}{1+\left(\frac{v_{1}}{v_{0}}\right)^{1-\frac{2 x}{l}}} ; \\
v_{z}=v_{x} \frac{h_{x}^{\prime}}{h_{x}} z=\frac{\left(v_{0}+v_{1}\right)}{1+\left(\frac{v_{1}}{v_{0}}\right)^{1-\frac{2 x}{l}} \cdot \frac{h_{x}^{\prime}}{h_{x}} z .}
\end{array}\right.
$$

According to the volume constant condition, i.e. $v_{0} h_{0} b_{0}=v_{1} h_{1} b_{1}, v_{1} / v_{0}=h_{0} b_{0} / h_{1} b_{1}=\xi$, then the velocity field can be simplified into the following form:

$$
\left\{\begin{array}{l}
v_{x}=\frac{(1+\xi) v_{0}}{1+\xi^{1-\frac{2 x}{l}}} ; \\
v_{y}=b_{x}^{\prime} \cdot v_{x}=\frac{\Delta b}{l} \cdot \frac{(1+\xi) v_{0}}{1+\xi^{1-\frac{2 x}{l}}} ; \\
v_{z}=v_{x} \frac{h_{x}^{\prime}}{h_{x}} z=\frac{(1+\xi) v_{0}}{2 x} \cdot \frac{h_{x}^{\prime}}{h_{x}} z . \xi^{1-\frac{2}{l}}
\end{array}\right.
$$

where, $v_{x}, v_{y}$ and $v_{z}$ are called the velocity components in the rolling direction, the spreading direction and the reduction direction, respectively. According to the geometric equation, we can get

$$
\left\{\begin{array}{l}
\dot{\varepsilon}_{x}=\frac{d v_{x}}{d x}=\frac{\left[2 v_{0}(1+\xi) \ln \xi\right] \xi^{\left(1-\frac{2 x}{l}\right)}}{l\left[1+\xi^{\left(1-\frac{2 x}{l}\right)}\right]^{2}}=\dot{\varepsilon}_{\max } \\
\dot{\varepsilon}_{y}=0 \\
\dot{\varepsilon}_{z}=-\dot{\varepsilon}_{\max }=-\frac{\left[2 v_{0}(1+\xi) \ln \xi\right] \xi^{\left(1-\frac{2 x}{l}\right)}}{l\left[1+\xi^{\left(1-\frac{2 x}{l}\right)}\right]^{2}}=\dot{\varepsilon}_{\min } .
\end{array}\right.
$$

where, $\dot{\varepsilon}_{x}, \dot{\varepsilon}_{y}$, and $\dot{\varepsilon}_{z}$ are the strain rate components in the rolling direction, the spreading direction and the reduction direction, respectively. The volume rate per second denoted by $U$ in the deformation zone can be written as follows:

$$
\begin{aligned}
U & =v_{x} h_{x} b_{x}=v_{n} h_{n} b_{n} \\
& =v_{R} \cos \alpha_{n}\left(R+h_{1}-R \cos \alpha_{n}\right)\left(b_{1}-\frac{\Delta b}{l} R \sin \alpha_{n}\right)=v_{1} h_{1} b_{1} .
\end{aligned}
$$

In Eqs 38, 39, due to $\dot{\varepsilon}_{x}+\dot{\varepsilon}_{z}=0 ; x=0, v_{x}=v_{0} ; x=l, v_{x}=v_{1}$; $z=0, v_{z}=0 ; z=h_{x}, v_{z}=-v_{x} \tan \alpha$, the velocity field meets the kinematically admissible condition rigorously and can be used as a basis for the following energy calculation.

\section{Internal Deformation Power}

It is known that the specific plastic power based on the Mises yield criterion is nonlinear, which is difficult to be integrated to obtain the internal deformation power. For solving this problem, a new method called the replacement method of specific plastic power is proposed here. This method is to replace the specific plastic power of the Mises yield criterion by the present specific plastic power based on the AS yield criterion. Therefore, the internal deformation power $N_{d}$ (Sezek et al., 2008) can be calculated by

$$
\begin{aligned}
N_{d} & =4 \int_{0}^{l} \int_{0}^{b_{m}} \int_{0}^{h_{x}} 0.577 \sigma_{s}\left(\dot{\varepsilon}_{\max }-\dot{\varepsilon}_{\min }\right) d x d y d z \\
& =4 \int_{0}^{l} \int_{0}^{b_{m}} \int_{0}^{h_{x}} 0.577 \sigma_{s} \cdot 2 \dot{\varepsilon}_{\max } d x d y d z \\
& =4.616 \sigma_{s} \int_{0}^{l} \int_{0}^{b_{m}} \int_{0}^{h_{x}} \frac{\left[2 v_{0}(1+\xi) \ln \xi\right] \xi^{\left(1-\frac{2 x}{l}\right)}}{l\left[1+\xi^{\left(1-\frac{2 x}{l}\right)}\right]^{2}} d x d y d z \\
& =4.616 \sigma_{s} U \cdot \ln \xi .
\end{aligned}
$$

\section{Friction Power}

According to the roller surface equation $d S=\sqrt{1+h_{x}^{\prime 2}} d x d y=$ $\sec \alpha d x d y$ and Eq. 36, the friction power consumed on the contact surface between the roller and the workpiece can be calculated by (Sezek et al., 2008) 


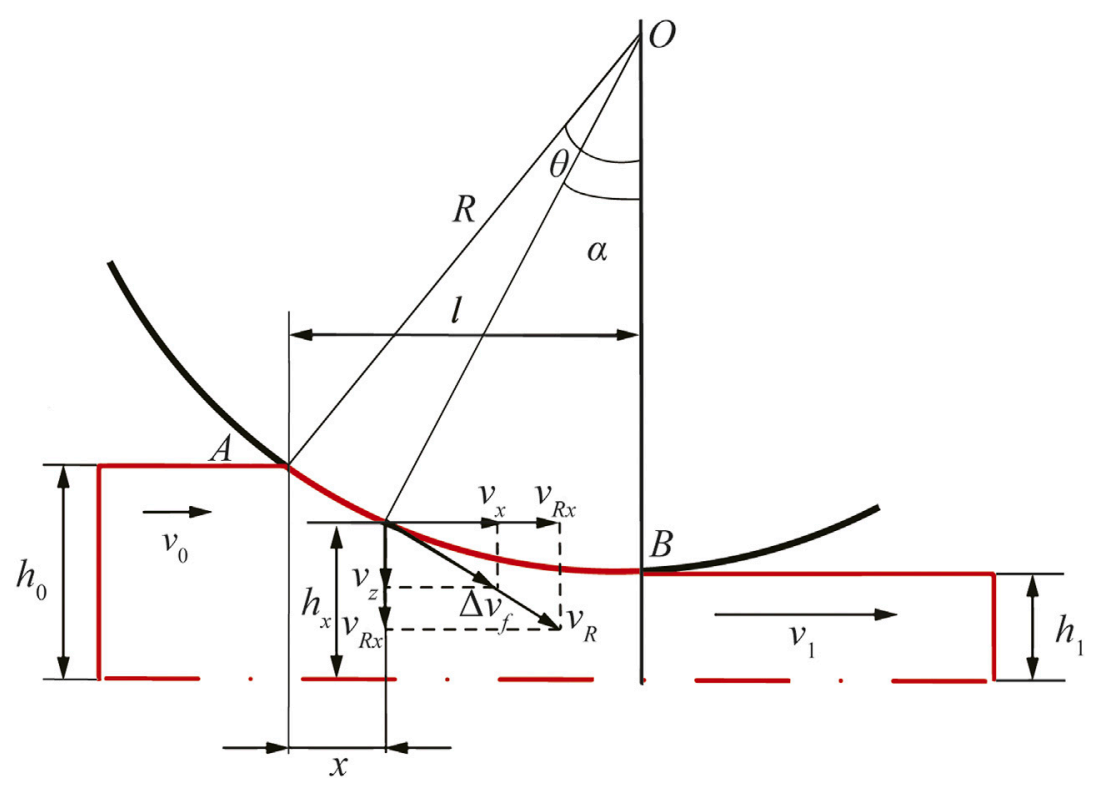

FIGURE 5 | The thick plate in the rolling deformation zone.

$$
\begin{aligned}
N_{f} & =4 m k \int_{0}^{l} \int_{0}^{b_{x}} \Delta v_{f} \sqrt{1+\left(h_{x}^{\prime}\right)^{2}} d x d y \\
\Delta v_{f} & =v_{R}-v_{x} \sqrt{1+\left(h_{x}^{\prime}\right)^{2}}=v_{R}-v_{x} \sec \alpha
\end{aligned}
$$

where, $m$ is the friction factor, $\Delta v_{f}$ is the velocity discontinuity. The shear yield strength is $k=\sigma_{s} / \sqrt{3}$. According to the collinear characteristic between the friction shear stress $\tau_{f}$ and the velocity discontinuity $\Delta v_{f}$, the shear power can be calculated by

$$
\begin{aligned}
N_{f} & =4 \int_{0}^{l} \int_{0}^{b_{x}} \tau_{f} \cdot \Delta v_{f} d S \\
& =4 \int_{0}^{l} \int_{0}^{b_{x}}\left(\tau_{f_{x}} \Delta v_{x}+\tau_{f_{y}} \Delta v_{y}+\tau_{f_{z}} \Delta v_{z}\right) \sqrt{1+\left(h_{x}^{\prime}\right)^{2}} d x d y \\
& =4 m k \int_{0}^{l} \int_{0}^{b_{x}}\left(\Delta v_{x} \cos \alpha+\Delta v_{y} \cos \beta+\Delta v_{z} \cos \gamma \sec \alpha\right) d x d y .
\end{aligned}
$$

From Figure 5, the direction cosines between $\Delta v_{f}$ and the coordinate axis are respectively

$$
\left\{\begin{array}{l}
\cos \alpha= \pm\left[R^{2}-(l-x)^{2}\right]^{1 / 2} / R \\
\cos \beta=0 \\
\cos \gamma= \pm(l-x) / R=\sin \alpha
\end{array}\right.
$$

Substituting Eq. 45 into Eq. 44, we can get

$$
\begin{aligned}
N_{f} & =4 m k\left[\int_{0}^{l} \int_{0}^{b_{x}}\left(v_{R} \cos \alpha-v_{x}\right) \cos \alpha \sec \alpha d x d y+\int_{0}^{l} \int_{0}^{b_{x}}\left(v_{R} \sin \alpha-v_{x} \tan \alpha\right) \cos \gamma \sec \alpha d x d y\right] \\
& =4 m k\left[\frac{\Delta b R^{2} v_{R}}{l}\left(1-2 \cos \alpha_{n}+\cos \theta\right)+R v_{R} b_{1}\left(\theta-2 \alpha_{n}\right)+\frac{R U}{h_{m}} \ln \frac{\tan ^{2}\left(\frac{\alpha_{n}}{2}+\frac{\pi}{4}\right)}{\tan \left(\frac{\theta}{2}+\frac{\pi}{4}\right)}\right] \\
& =4 m k b_{1}\left[R v_{R}\left(\theta-2 \alpha_{n}\right)+\frac{R U}{b_{1} h_{m}} \ln \frac{\tan ^{2}\left(\frac{\alpha_{n}}{2}+\frac{\pi}{4}\right)}{\tan \left(\frac{\theta}{2}+\frac{\pi}{4}\right)}\right] .
\end{aligned}
$$

where, $h_{m}$ is the average value of $h_{x}$.

\section{Shearing Power}

From Eqs 35, 37, we can see that: on the exit section $x=l$, $h_{x}^{\prime}=b_{x}^{\prime}=0 ;\left.v_{y}\right|_{x=l}=\left.v_{z}\right|_{x=l}=0$ is on the exit section. Therefore, we can have

$$
\left|\bar{v}_{z}\right|_{x=0}=\sqrt{\bar{v}_{y}^{2}+\left.\bar{v}_{z}^{2}\right|_{x=0}}
$$

Since there is no shear power consumed on the outlet section, the shear power $N_{s}$ consumed on the inlet section is equal to the total shear power. Therefore, we have

$$
\begin{aligned}
N_{s} & =4 \int_{0}^{h_{0}} \int_{0}^{b_{0}} \sqrt{\bar{v}_{y}^{2}+\bar{v}_{z}^{2}} d y d z \\
& =4 k \int_{0}^{h_{0}} \int_{0}^{b_{0}} \frac{v_{0} \Delta b}{l} \sqrt{1+\frac{l^{2} \tan ^{2} \theta}{4 \Delta b^{2}}} d y d z \\
& =\frac{2 k U}{l} \sqrt{4 \Delta b^{2}+l^{2} \tan ^{2} \theta} .
\end{aligned}
$$

\section{Total Power of Rolling Deformation and its Extreme Value}

The total power $\Phi$ can be calculated by

$$
\Phi=N_{d}+N_{s}+N_{f}
$$

Therefore, by adding Eqs 41, 46, 48, it results in

$$
\Phi=4.616 \sigma_{s} U \cdot \ln \xi+\frac{2 k U \Delta h}{l}+4 m k b_{1}\left[R v_{R}\left(\theta-2 \alpha_{n}\right)+\frac{R U}{b_{1} h_{m}} \ln \frac{\tan ^{2}\left(\frac{\alpha_{n}}{2}+\frac{\pi}{4}\right)}{\tan \left(\frac{\theta}{2}+\frac{\pi}{4}\right)}\right]
$$


TABLE 1 | Validation of the predictive models of rolling force and torque with measured data.

\begin{tabular}{|c|c|c|c|c|c|c|c|c|c|c|c|c|}
\hline Pass No & $\begin{array}{c}\mathbf{v}_{\mathbf{R}} \\
\mathbf{m} \cdot \mathbf{s}^{-1}\end{array}$ & $\begin{array}{c}\mathbf{T} \\
\rho^{\circ} \mathrm{C}\end{array}$ & $\begin{array}{l}2 \mathrm{~h}_{0} \\
/ \mathrm{mm}\end{array}$ & $\begin{array}{c}2 h_{1} \\
/ \mathrm{mm}\end{array}$ & $\begin{array}{c}2 b_{0} \\
/ \mathrm{mm}\end{array}$ & $\begin{array}{c}2 b_{1} \\
/ \mathrm{mm}\end{array}$ & $\begin{array}{c}F_{M} \\
/ \mathbf{k N}\end{array}$ & $\begin{array}{c}F_{\mathrm{A}} \\
/ \mathbf{k N}\end{array}$ & $\begin{array}{l}\Delta_{1} \\
/ \%\end{array}$ & $\begin{array}{c}M_{\mathrm{M}} \\
/ \mathrm{kN} \cdot \mathrm{m}\end{array}$ & $\begin{array}{c}M_{\mathrm{A}} \\
/ \mathrm{kN} \cdot \mathrm{m}\end{array}$ & $\begin{array}{l}\Delta_{2} \\
/ \%\end{array}$ \\
\hline 2 & 1.64 & 945 & 299 & 272 & $3,472.0$ & $3,474.4$ & 43,607 & 45,073 & 3.4 & 2,640 & 2,740 & 3.8 \\
\hline 3 & 1.66 & 933 & 272 & 245 & $3,474.4$ & $3,476.6$ & 44,006 & 45,407 & 3.2 & 2,694 & 2,774 & 3.0 \\
\hline 4 & 1.68 & 923 & 245 & 219 & $3,476.6$ & $3,478.6$ & 43,172 & 46,936 & 8.7 & 2,665 & 2,895 & 8.6 \\
\hline 5 & 1.82 & 925 & 219 & 194 & $3,478.6$ & $3,480.5$ & 42,269 & 47,675 & 12.8 & 2,430 & 2,733 & 12.5 \\
\hline 6 & 1.97 & 932 & 194 & 173 & $3,474.4$ & $3,482.0$ & 39,061 & 40,565 & 3.9 & 2,101 & 2,174 & 3.4 \\
\hline
\end{tabular}

From Eqs 40, 41, 46, 48, one can obtain that

$$
\begin{gathered}
\frac{d U}{d \alpha_{n}}=v_{R} b_{1} R\left(\sin 2 \alpha_{n}-\frac{R+h_{1}}{R} \sin \alpha_{n}\right) \\
-\frac{\Delta b}{l} R^{2} v_{R}\left(\frac{R+h_{1}}{R} \cos 2 \alpha_{n}+\sin 2 \alpha_{n}-\cos ^{3} \alpha_{n}\right)=N \\
\frac{d N_{d}}{d \alpha_{n}}=4.616 \sigma_{s} N \cdot \ln \xi \\
\frac{d N_{s}}{d \alpha_{n}}=\frac{2 k N}{l} \sqrt{4 \Delta b^{2}+l^{2} \tan ^{2} \theta} \\
\frac{d N_{f}}{d \alpha_{n}}=4 m k\left[\frac{N R}{h_{m}} \ln \frac{\tan ^{2}\left(\frac{\pi}{4}+\frac{a_{n}}{2}\right)}{\tan \left(\frac{\pi}{4}+\frac{\theta}{2}\right)}+\frac{2 U R}{h_{m} \cos a_{n}}-2 v_{R} b_{1} R\right] .
\end{gathered}
$$

Therefore, we can have

$$
\frac{d \Phi}{d \alpha_{n}}=\frac{d N_{d}}{d \alpha_{n}}+\frac{d N_{s}}{d \alpha_{n}}+\frac{d N_{f}}{d \alpha_{n}} .
$$

When the total power $\Phi$ achieves the minimum value, the theoretical expression of the friction factor $m$ can be obtained by solving Eq. 55. Therefore, we have

$$
m=\frac{-N\left(4.616 \sigma_{s} \ln \xi+\frac{2 k}{l} \sqrt{4 \Delta b^{2}+l^{2} \tan ^{2} \theta}\right)}{4 k\left[\frac{N R}{h_{m}} \ln \frac{\tan ^{2}\left(\frac{\pi}{4}+\frac{a_{n}}{2}\right)}{\tan \left(\frac{\pi}{4}+\frac{\theta}{2}\right)}+\frac{2 U R}{h_{m} \cos a_{n}}-2 v_{R} b_{1} R\right]}
$$

Substituting a neutral angle $\alpha_{n}$ into Eq. 56, then the friction factor $m$ can be obtained. Therefore, we can obtain the analytical solution of rolling torque $M$, rolling force $F$ and stress state coefficient $n_{\sigma}$ :

$$
M=\frac{R}{2 v_{R}} \Phi_{\min } ; F=\frac{M}{\chi \sqrt{2 R \Delta h}} ; n_{\sigma}=\frac{F}{4 b l k}
$$

where, the arm coefficient $\chi$ can refer to the literature (Harris, 2014).

From the above derivation, it can be seen that the present new method of using a linear yield criterion to analyze a new velocity field can solve the problem of integral difficulty due to the

TABLE 2 | Comparison of the predicted outlet thickness with the measured one.

\begin{tabular}{lccccc} 
Pass No & $\boldsymbol{F}_{\mathbf{M}} \mathbf{~ k N}$ & $\boldsymbol{S}_{\boldsymbol{0}} \mathbf{~} \mathbf{m m}$ & $\mathbf{2 h}_{\boldsymbol{1}} \mathbf{/ m m}$ & $\boldsymbol{H}_{\boldsymbol{f}} / \mathbf{m m}$ & $\boldsymbol{\Delta}_{\mathbf{H}} / \mathbf{m m}$ \\
\hline 2 & 43,607 & 267.95 & 272.02 & 272.16 & 0.14 \\
3 & 44,006 & 241.26 & 245.37 & 245.50 & 0.13 \\
4 & 43,172 & 214.76 & 218.80 & 219.15 & 0.35 \\
5 & 42,269 & 189.92 & 193.87 & 194.37 & 0.50 \\
6 & 39,061 & 169.52 & 173.17 & 173.31 & 0.14
\end{tabular}

nonlinear Mises yield criterion and can provide the possibility of acquiring an analytical model of rolling force for multicomponent alloys. It also can be seen that such a model has a clear functional relationship, which is convenient to be used online in the process parameter design and the thickness control of a rolled plate.

\section{Experimental Validation of Rolling Force and Thickness Prediction}

An on-site rolling experiment was carried out in a domestic factory. The roller diameter is $1120 \mathrm{~mm}$, and the billet size is $320 \mathrm{~mm} \times 2050 \mathrm{~mm} \times 3250 \mathrm{~mm}$. The measured rolling velocities for the 2 nd-6th passes are $1.64,1.66,1.68,1.82$, and $1.97 \mathrm{~m} / \mathrm{s}$ respectively. The arm coefficient $\chi$ is respectively taken as $0.49,0.50,0.51,0.49$, and 0.50 ; the corresponding rolling temperatures are $944.56^{\circ} \mathrm{C}, 933.49^{\circ} \mathrm{C}, 922.97^{\circ} \mathrm{C}$, $924.68^{\circ} \mathrm{C}$, and $932.11^{\circ} \mathrm{C}$, respectively. The outlet thickness and the rolling force for each pass can be accurately acquired by thickness measurement. The deformation resistance of the multicomponent alloys in the experiment adopted in the present paper is (Zhang, 2018)

$$
\begin{aligned}
& \sigma_{s}=3583.195 \mathrm{e}^{-2.233 \times 10^{-3} T} \cdot \varepsilon^{0.42437} \cdot \dot{\varepsilon}^{-0.3486 \times 10^{-3} T+0.46339} ; \\
& T=t+273 .
\end{aligned}
$$

where, $\varepsilon$ is effectiveness strain, $\dot{\varepsilon}$ is effectiveness strain rate, $t$ is deformation temperature, $T$ is Kelvin temperature.

The rolling torque and rolling force for the above passes can be predicted by Eq. 57, which is shown in Table 1 .

In Table 1, $\Delta_{1}$ denotes the relative error between the calculated rolling force $F_{A}$ and the experimental one $F_{M}$. It can be seen that the maximum error is $12.8 \% . \Delta_{2}$ denotes the relative error between the calculated rolling torque $M_{A}$ and the experimental one $M_{M}$. As shown in Table 1, the maximum error is $12.5 \%$. Therefore, both the two maximum errors are within the engineering allowable value of 15\% (Wang et al., 2019). It should be noted here that it is better to further give the comparison between the present results based on the present yield criterion and those based on the other yield criteria, which can further reflect the superiority of the present yield criterion. However, due to the complexity of giving a set of formulas based on the other yield criteria synchronously, it is inconvenient to do this work at this time. In the future, we will work on this aspect systematically.

At the same time, the rolling force model obeys the bounce equation (Sun et al., 2013) 


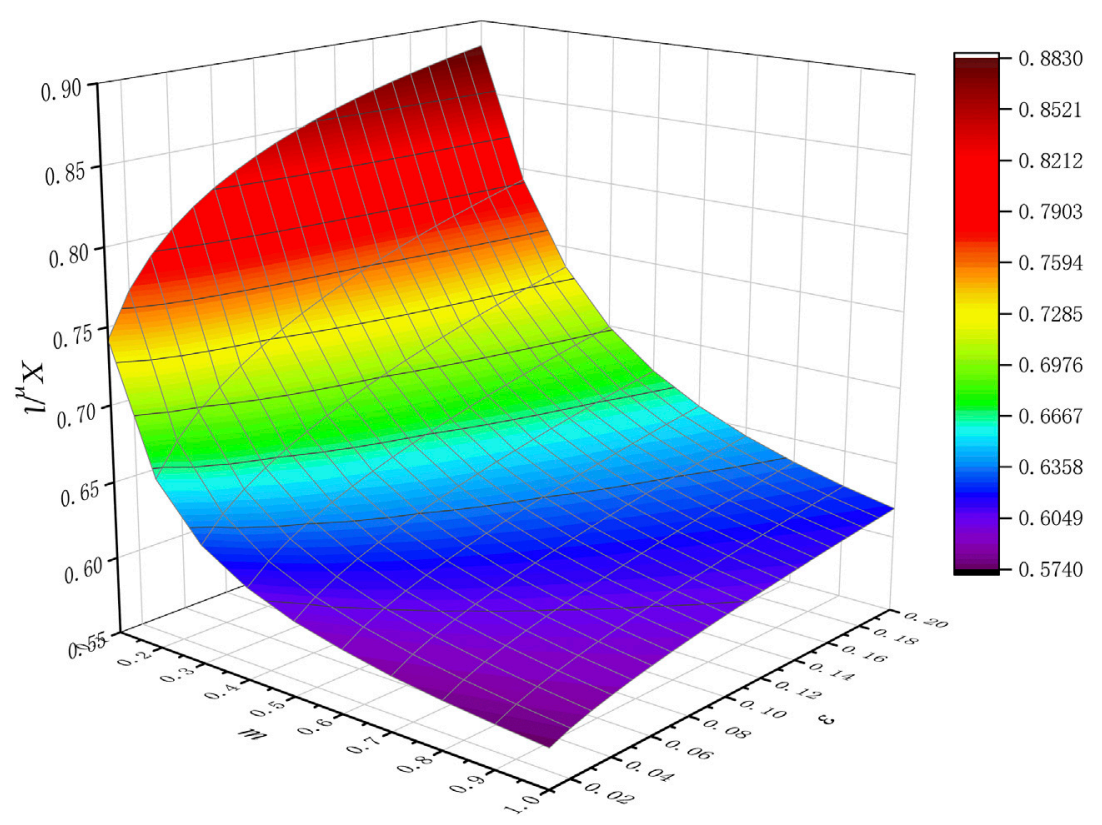

FIGURE 6 | Effect of friction factor and relative reduction on the neutral point position.

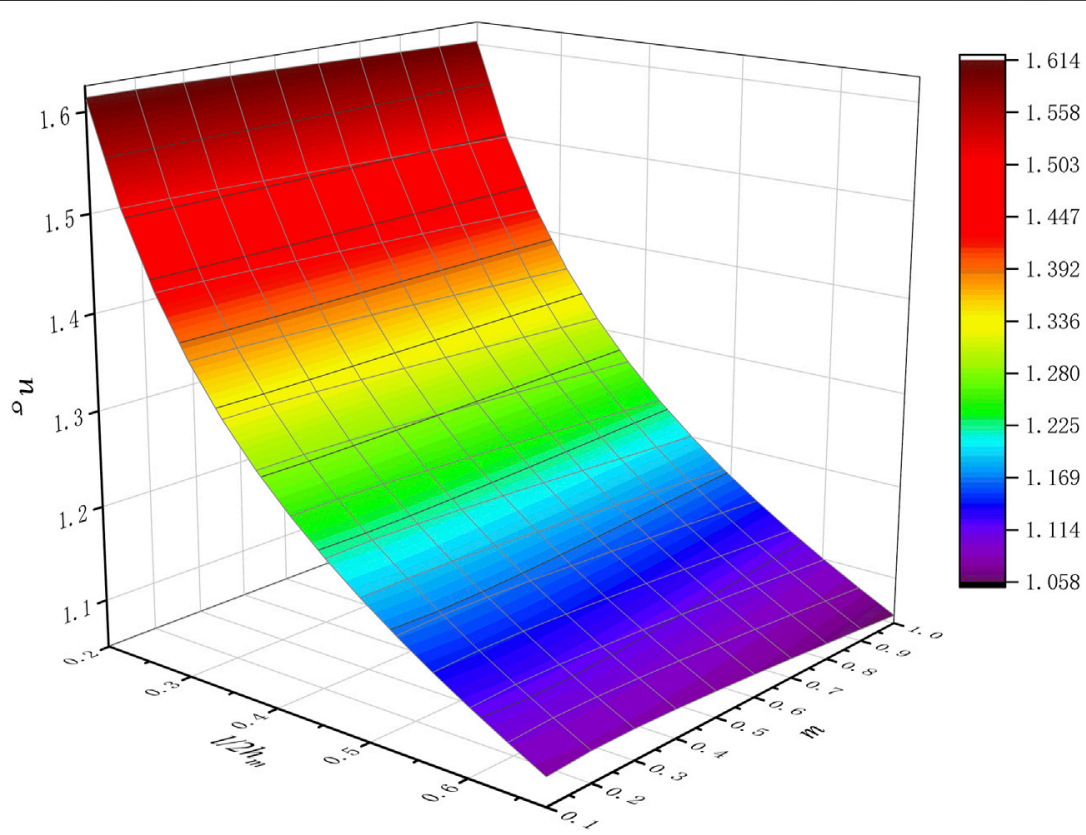

FIGURE 7 | Effect of the shape factor and friction factor on the stress state coefficient.

$$
H_{f}=S_{0}+E=S_{0}+\frac{P}{K}
$$

where, $H_{f}$ is the final thickness of the rolled product, $S_{0}$ is the noload roll gap which can be determined by the regression of experimental data of measured rolling force and measured exit thickness in Table 1, $E$ is the bouncing amount of the rolling mill, $P$ is the rolling force, and $K$ is the stiffness coefficient of the rolling mill, $K=10700 \mathrm{kN} / \mathrm{mm}$ is for the present rolling mill.
Based on Eq. 59, the comparison between the predicted value of outlet thickness and the measured value for each pass can be shown in Table 2.

In Table 2, $\Delta_{H}$ is the absolute error between the measured exit thickness $2 h_{1}$ and the predicted exit thickness $H_{f}$. The average error remains at $0.25 \mathrm{~mm}$, and the maximum error does not exceed $0.50 \mathrm{~mm}$, which meets the allowable deviation of $\mathrm{GB} / \mathrm{T}$ 709-2019. Therefore, it can be concluded that the prediction 


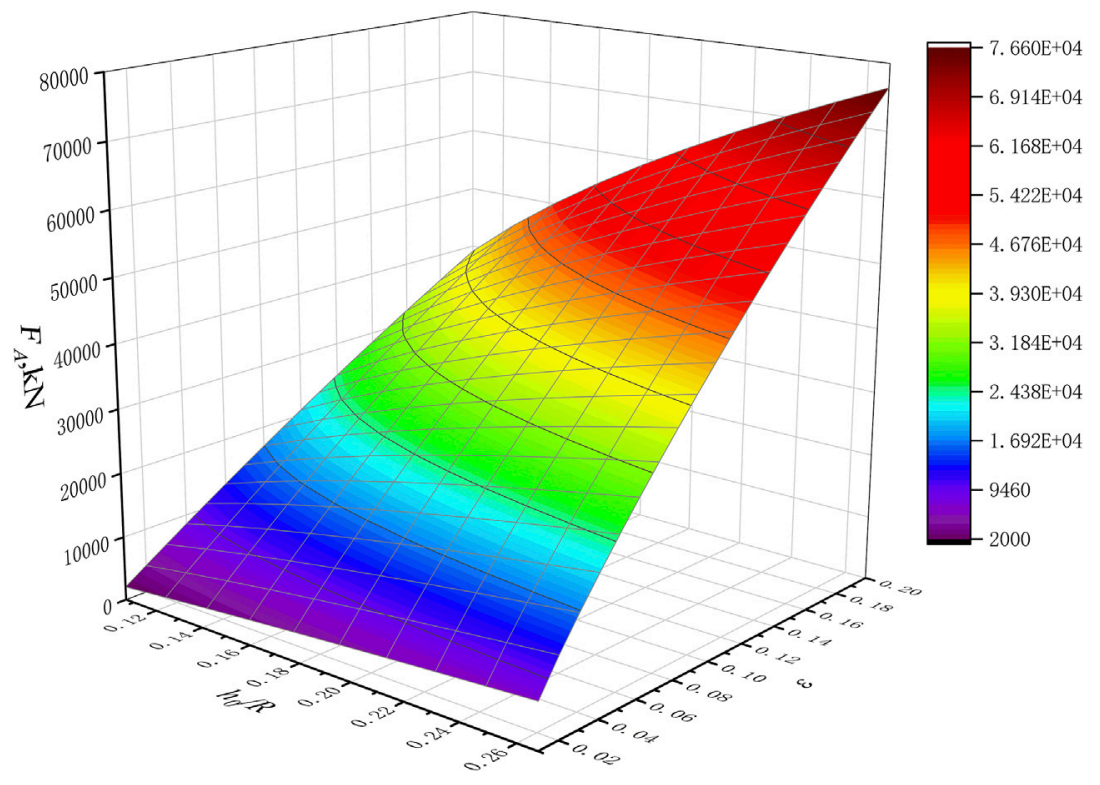

FIGURE 8 | Effect of the thickness to radius ratio and relative reduction on the rolling force.

accuracy is high. Also, it can be concluded that due to the high precision of the rolling force model, the precision of the outlet thickness is also high, which can provide scientific guidance for rolling process optimization.

\section{ANALYSIS AND DISCUSSION}

Figure 6 shows the effect of friction factor $m$ and relative reduction $\varepsilon$ on the neutral point position $X_{n} / l$. It is seen that with the increase of $m$, the position of the neutral point gradually moves towards the entrance, while with the increase of $\varepsilon$, the position of the neutral point moves towards the exit.

Figure 7 shows the effect of shape factor $l / 2 h_{m}$ and the friction factor $m$ on the stress state coefficient $n_{\sigma}$. It can be seen that as the shape factor increases, the $n_{\sigma}$ decreases significantly. The friction factor $m$ has a small effect on $n_{\sigma}$, indicating that the friction power has little influence on the total power.

Figure 8 shows the effect of the thickness-radius ratio $h_{0} / R$ and the reduction ratio $\varepsilon$ on the calculated rolling force $F_{A}$. It can be seen that with the increase of the reduction ratio and the increase of the ratio of thickness to radius, the rolling force increases.

From the above comparison and analysis, it should be noted here that by comparing with the previous method, the present method of using a new yield criterion to analyze the present velocity field can not only provide an analytical solution of rolling force rather than a numerical one, but also can give more reasonable results with high precision (within 15\%). This is due to the effectiveness of approximating to the Mises yield criterion with the present linear one and the rationality of proposing the present velocity field in the form of logistic function. In a word, the present paper has proposed a new method of analyzing the plate rolling process for multi component alloys. Based on this superiority, the process parameters of plate rolling can be designed and the thickness of the rolled plate can be controlled well, and the changing tendency of the rolling force along with many process parameters, such as the reduction, friction factor, thickness-toradius ratio, can be disclosed quantitatively.

\section{CONCLUSION}

1) The AS yield criterion proposed in this paper is a linear combination of principal stress components. Its yield locus is an equilateral and non-equiangular dodecagon with side length of $0.423 \sigma_{s}$. It intersects with the Mises circle, whose vertex angles are $150.71^{\circ}$ and $149.29^{\circ}$ respectively. The calculated results of the AS yield criterion are in good agreement with the experimental data, and the linear specific plastic power that lies in between the one based on the Tresca criterion and the one based on the TSS criterion is also derived.

2) This paper establishes a new velocity field based on the logistic function. This velocity field is proved to be satisfy the kinematically admissible condition rigorously. Based on the velocity field, the analytical solutions of rolling force and torque are established. After comparing with the measured rolling data, it is found that the analytical ones are in good agreement with the actual measured ones. The maximum errors for the rolling force and torque are 12.8 and $12.5 \%$ respectively. Besides, the bounce equation that based on the rolling force model is also given, and it is found that the predictive error is maintained at $0.25 \mathrm{~mm}$, and the maximum error is no more than $0.50 \mathrm{~mm}$. It can be seen that the method of using the AS yield criterion to analyze the proposed velocity field in this paper is effective. 
3) It is found that as the friction factor $m$ increases, the neutral point moves toward the inlet. As the reduction rate $\varepsilon$ increases, the neutral point moves toward the outlet. The stress state coefficient will decrease with the increase of the shape factor $l / 2 h_{m}$, and the increase in the thickness-to-radius ratio $h_{0} / R$ of the rolled plate will cause an increase in the rolling force.

\section{DATA AVAILABILITY STATEMENT}

The original contributions presented in the study are included in the article/Supplementary Material, further inquiries can be directed to the corresponding author.

\section{REFERENCES}

Drucker, D. C., and Prager, W. (1952). Soil Mechanics and Plastic Analysis or Limit Design. Quart. Appl. Math. 10 (2), 157-165. doi:10.1090/qam/48291

Gao, J. P., and Yu, M. H. (2005). Study on the Unified Yield Criterion of ThreeShear Stress [J]. J. Xi'an Univ. Architecture Technol. Nat. Sci. Edition 37 (4). doi:10.15986/j.1006-7930.2005.04.019

Harris, J. N. (2014). Mechanical Working of Metals: Theory and practice[M], Vol. 36. Oxford: Elsevier.

Hosford, W. F., and Caddell, R. M. (2011). Metal Forming: Mechanics and metallurgy $[M]$. New York: Cambridge University Press.

Jia, W. T., Ma, L. F., and Ma, Z. Y. (2016). As-cast Rolling Process and Flow Stress of AZ31B Magnesium Alloy[J]. Rare Metal Mater. Eng. (1), 152-158.

Kobayashi, S., Kobayashi, S., and Oh, S. I. (1989). Metal Forming and the FiniteElement method[M]. Oxford: Oxford University Press on Demand.

Lessells, J. M., and Macgregor, C. W. (1940). Combined Stress Experiments on a Nickel-Chrome-Molybdenum Steel. J. Franklin Inst. 230 (2), 163-181. doi:10.1016/s0016-0032(40)90729-3

Li, S., Wang, Z., Liu, C., Ruan, J., and Xu, Z. (2017). A Simplified Method to Calculate the Rolling Force in Hot Rolling[J]. Int. J. Adv. Manufacturing Tech. 88 (5-8), 2053-2059. doi:10.1007/s00170-016-8890-z

Li, T., and Wang, Z. W. (1996). Characteristic Field of Plane Stress Problem for the Orthotropic Materials[J]. J. Harbin Inst. Tech. (04), 123-126.

Liu, Y. M. (2017). Research on Mathematical Model of Strip Rolling Force and Shape Control Based on Energy Method [D]. Shenyang: Northeastern University.

Lode, W. (1926). Versuche über den Einfluß der mittleren Hauptspannung auf das Fließen der Metalle Eisen, Kupfer und Nickel[J]. Z. für Physik A Hadrons Nuclei 36 (11), 913-939. doi:10.1007/bf01400222

Maxey, W. A. (1974). Measurement of Yield Strength in the Mill Expander[C]. Proc. fifth Symp. line pipe Res., 20-22.

Mises, R. V. (1913). Mechanik der festen Körper im plastisch-deformablen Zustand [J]. Nachrichten von der Gesellschaft der Wissenschaften zu Göttingen. Mathematisch-Physikalische Klasse 1913 (4), 582-592.

Mises, R. V. (1928). Mechanik der plastischen Formänderung von Kristallen. $Z$. Angew. Math. Mech. 8 (3), 161-185. doi:10.1002/zamm.19280080302

Mohr, O. (1900). Welche Umstände bedingen die Elastizitätsgrenze und den Bruch eines Materials[J]. Z. des Vereins Deutscher Ingenieure 46 (1524-1530), 1572-1577.

Naghdi, P. M., Essenburg, F., Jr, and Koff, W. (1957). An Experimental Study of Initial and Subsequent Yield Surfaces in plasticity[J]. New York: Mechanics Reviews, 201-209.

Sezek, S., Aksakal, B., and Can, Y. (2008). Analysis of Cold and Hot Plate Rolling Using Dual Stream Functions. Mater. Des. 29 (3), 584-596. doi:10.1016/ j.matdes.2007.03.005

\section{AUTHOR CONTRIBUTIONS}

SHZ: Responsible for modeling of rolling force; JLX: Responsible for collection of experimental data and model validation; LZC: Responsible for grammar modification.

\section{FUNDING}

This work is funded by the National Natural Science Foundation of China (Grant No. 52074187, U1960105, 51504156) and the Natural Science Foundation of Jiangsu Province (Grant No. BK20180095).

Sun, J., Peng, Y., and Liu, H. (2013). Coupled Dynamic Modeling of Rolls Model and Metal Model for Four High Mill Based on Strip crown Control. Chin. J. Mech. Eng. 26 (1), 144-150. doi:10.3901/cjme.2013.01.144

Tresca, H. (1864). Memoir on the Flow of Solid Bodies under strong Pressure[J]. Comptes-rendus de l'académie des Sci. 59, 754-758.

Wang, Z. F. (2000). Application of 3D Fluxional Function to Metal Forming [J]. J. Harbin Inst. Tech. (05), 93-96.

Wang, Z., Zhang, D., Gong, D., and Peng, W. (2019). A New Data-Driven Roll Force and Roll Torque Model Based on FEM and Hybrid PSO-ELM for Hot Strip Rolling. ISIJ Int. 59 (9), 1604-1613. doi:10.2355/isijinternational.isijint-2018-846

Yu, M. H., He, L. N., and Song, L. Y. (1985). Twin Shear Stress Theory and its Generalization[J]. Sci. China Ser. A-Mathematics, Phys. Astron. Technol. Sci. 28 (11), 1174-1183.

Yu, M.-h. (2002). Advances in Strength Theories for Materials under Complex Stress State in the 20th century. Appl. Mech. Rev. 55 (3), 169-218. doi:10.1115/1.1472455

Zhang, S. H. (2018). Linearization of Yield Criterion and its Engineering applications $[M]$. Beijing: Metallurgical Industry Press.

Zhang, S. H., Deng, L., Zhang, Q. Y., Li, Q. H., and Hou, J. X. (2019). Modeling of Rolling Force of Ultra-heavy Plate Considering the Influence of Deformation Penetration Coefficient. Int. J. Mech. Sci. 159, 373-381. doi:10.1016/j.jimecsci.2019.05.048

Zhang, S. H., Gao, S. W., Wu, G. J., Cao, J., and Zhao, D. W. (2017). A Weighted Average Yield Criterion and its Applications to Burst Failure of Pipeline and Three-Dimensional Forging. J. Manufacturing Process. 28, 243-252. doi:10.1016/j.jmapro.2017.06.006

Zhang, Y. F., Di, H. S., Li, X., Peng, W., Zhao, D-W., and Zhang, D-H. (2020). A Novel Approach for the Edge Rolling Force and Dog-Bone Shape by Combination of Slip-Line and Exponent Velocity Field[J]. SN Appl. Sci. 2 (12), 1-11. doi:10.1007/s42452-020-03770-3

Conflict of Interest: The authors declare that the research was conducted in the absence of any commercial or financial relationships that could be construed as a potential conflict of interest.

Publisher's Note: All claims expressed in this article are solely those of the authors and do not necessarily represent those of their affiliated organizations, or those of the publisher, the editors and the reviewers. Any product that may be evaluated in this article, or claim that may be made by its manufacturer, is not guaranteed or endorsed by the publisher.

Copyright $\odot 2021$ Zhang, Xin and Che. This is an open-access article distributed under the terms of the Creative Commons Attribution License (CC BY). The use, distribution or reproduction in other forums is permitted, provided the original author(s) and the copyright owner(s) are credited and that the original publication in this journal is cited, in accordance with accepted academic practice. No use, distribution or reproduction is permitted which does not comply with these terms. 